\title{
Precise Geometry Simulation of Interferometric SAR Signal for Air- and Spaceborne Sensors
}

\author{
Marc Bara (1), Antoni Broquetas (2), Josep Closa (3)
}

\author{
(1) Universitat Politècnica de Catalunya (UPC). C/ Jordi Girona, 1-3, E-08034 Barcelona, Spain. \\ Phone / Fax: +3493 4011065 / +34 93401 7232. E-mail: mabara@tsc.upc.es \\ (2) Institut de Geomàtica, Parc de Montjuïc, E-08038 Barcelona, Spain. \\ Phone / Fax: +34 935671500 / +34 93567 1567. E-mail: abroquetas@icc.es \\ (3) ESA/ESRIN, Via Galileo Galilei, I-00044 Frascati, Italy. \\ Phone / Fax: +3906941801/+390694180 280. E-mail: josep.closa@esrin.esa.it
}

\begin{abstract}
A complete interferometric Synthetic Aperture Radar (SAR) simulator has been developed. It achieves precise results by using geodetic models to represent the Earth's shape, and considering SAR effects such as range migration, Doppler shifts, or perturbations of movement in the airborne case, which are included via the inverse Extended Chirp Scaling Algorithm (ECSA).
\end{abstract}

\section{INTRODUCTION}

SAR raw signal simulation, its problems and its advantages in regard to the optimization of SAR system parameters and the development and validation of new processing and interferometric algorithms have been widely discussed in the literature (e.g, [1]). To sum up, simulation allows the control of a great variety of, not only system configurations, (sensor trajectories, PRFs, squint angles...) but also of scene characteristics (roughness, slope, permitivity...) in a parametric manner. In this paper a complete simulation scheme is presented, which is able to deal with all kind of SAR sensors, not only spaceborne as considered traditionally, but also airborne by means of the inclusion of its own differential characteristics.

\section{GEOMETRICAL AND SURFACE MODEL}

Many geometrical models can be selected to simulate a SAR mission. Options with an easy solution, such as flat or spherical Earth, are good enough in several situations in which a higher precision is not required. However, for this simulator a more accurate model has been chosen: the elliptical model. This model allows naturally the use of standard cartographic coordinates systems, and thus the results can be easily related to geodesic coordinates [4].

The main input of a simulator, from the geometrical point of view, should the platform position for every point in the space from where the system has transmitted a pulse. In this simulator some possibilities have been implemented. On the one hand, for the spaceborne case it is possible to provide the algorithm with orbit information given by five state vectors (as the Precise Orbit files of the ERS-1/2 satellites), which include position and velocity components at equally spaced mission times. Starting from these state vectors, position data at every azimuth time is obtained by means of interpolation.
On the other hand, in the airborne case the procedure accepts two values as an input: a constant altitude over the ellipsoid and the heading with respect to the north, since these two values are enough to represent a nominal trajectory. One important remark is the following: an airborne simulator must be designed to include the coordinates of a true flight, with possible deviations of position and attitude from the nominal straight track. In our implementation this has been done during the inverse SAR processing (see next section), by means of the inclusion of several steps to distort the nominal impulse response. Therefore, the airborne slant-range reflectivity will simulate a correctly focused image, while the effect of platform deviations has been included in the second part of the algorithm. Indeed, this solution can be considered the most accurate one, since the real airborne raw data are not distorted in the image domain but in the signal domain.

Regarding the information to characterize the terrain itself, the required data is the digital elevation map (DEM), as well as the electromagnetic characteristics of the terrain which is being simulated (permitivity $\varepsilon$ or conductivity $\sigma$ ).

Once the Earth's shape and the SAR location are modeled, an important stage is to calculate each facet's vertex position. This can be done for every position of the trajectory by defining a plane in the looking direction, perpendicular to the forward axis (zero-Doppler geometry). Its intersection with the Earth is an ellipse that contains all facets' vertexes in the range direction.

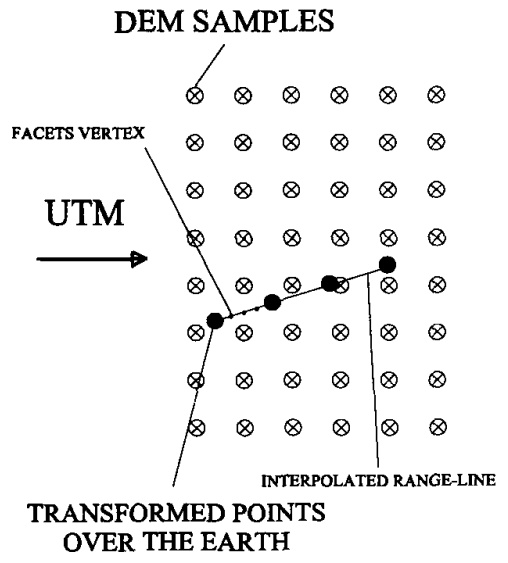

Fig. 1 Vertex location within the UTM grid 
This ellipse, projected to the UTM plane, follows a nearly straight line where all height data is available (Fig. 1). In this process it is necessary to account for the transformations from values given in a three-dimensional Cartesian axis to UTM coordinates using the appropriate formulation [4].

The SAR sensor moves to the next position in azimuth and the operation is repeated. Every range-line is assumed independent as if the SAR was sensing in a start-stop mode. When every vertex coordinates are known, their height and permitivity values can be assigned interpolating the grid data.

The next point to consider is the electromagnetic characterization of the individual facets, which has been based on a surface model assuming a backscatter far-field solution using physical optics. Finally, the simulator also includes the effect of the speckle noise, which has been considered by adding a Gaussian distribution to the height values of the scene, so that its summation results in a Rayleigh distribution on the intensity image [1].

\section{GENERATION OF SAR RAW DATA}

Once the reflectivity map of the terrain is obtained, the following step is to generate the SAR raw data. In order to emulate the SAR impulse response of a scatter situated in any point of the swath, we can consider to use methods of a SAR processing algorithm in an inverse way. In this simulator an inverse version of the ECSA has been implemented.

The ECSA algorithm is widely described in [3], thus, in this paper we will only explain the main differences. Basically, in this implementation we have used the conjugate functions of the standard algorithm in an inverse order, considering the appropriate curvature factor, effective chirp rate, secondary range compression along with other parameters. An important difference between the simulation and the processing schemes is the motion compensation. In general, the motion is compensated by the calculation of a phase term which represents the variation between the nominal and the real position of the antenna on the line of sight direction, also called LOS (line-of-sight) displacement. From the processor point of view, this phase term is applied twice, before the range compression for a reference range distance (first order motion compensation) and once the signal is focused in range (second order), which ensures the correct positioning of the compressed signal.

In our implementation, the following functions for motion compensation are considered:

$$
\begin{aligned}
& \Phi_{m c_{-} 1}\left(t, f ; r_{r e f}\right)=\exp \left[\frac{-4 \pi}{\lambda} j \cdot \Delta r_{p e r p}\left(t, r_{r e f}\right) \cdot\left(1+\frac{\lambda f}{c}\right) \cdot \cos \left(\beta_{r e f}\right)\right] \\
& \begin{array}{c}
\Phi_{m c_{-2} 2}(t, r)=\exp \left[\frac { - 4 \pi } { \lambda } j \cdot \left(\Delta r_{\text {perp }}(t, r) \cos (\beta(r))-\right.\right. \\
\left.\left.\Delta r_{\text {perp }}\left(t ; r_{\text {ref }}\right) \cos \left(\beta_{\text {ref }}\right)\right)\right]
\end{array}
\end{aligned}
$$

where $\lambda$ is the wavelength, $c$ the speed of light, $t$ is the azimuth time, $f$ the range frequency, $r$ the distance to the target, $r_{r e f}$ is the reference range distance, $\beta(r)$ is the squint angle as a function of range and $\beta_{\text {ref }}$ is the squint angle for the reference distance. As it can be noticed, (1) is applied in the range frequency domain, to compensate not only the phase but also the modulus position for a given reference distance. Equation (2) is employed to account for the range variation of the movement. They both contain the function $\Delta r_{p e r p}(t, r)$, which is the deviation of the sensor with respect to the nominal straight track, measured in the slant range zeroDoppler plane. It can be approximated by:

$$
\Delta r_{p e r p}(t, r) \approx \Delta y(t) \cdot \sin \left(\theta_{L}\right)-\Delta z(t) \cdot \cos \left(\theta_{L}\right)
$$

where the symbols are defined as in Fig. 2. The presence of the squint angle is included in the geometry and, thus, the correct line of sight distance is given by $\Delta r_{p e r p}(t, r)$ multiplied by $\cos (\beta(r))$, as appears in (1) and (2).

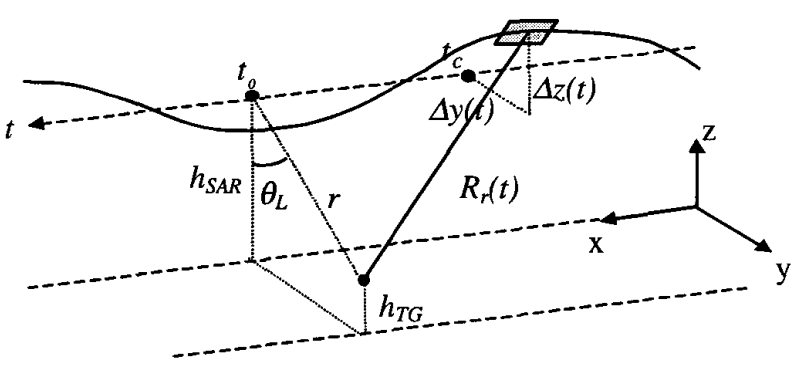

Fig. 2. Airborne SAR geometry

The problem of the SAR processor is that it must apply (1)(3) without having a precise knowledge of the look angle $\theta_{L}$, since it depends on the unknown target's height $h_{T G}$. As a result of this, a reference height must be used and some errors can be introduced [5]. However, from the simulation point of view, the second-order compensation is applied at the initial stage, and the terrain relief which is being simulated is known perfectly. Therefore, all the functions can be employed without flat-Earth approximations.

\section{INTERFEROMETRIC SIMULATOR}

There are two main techniques to simulate an interferogram. It can be obtained as the phase difference between two images, being each one the output of a simulator as the one described above. Another way consists of evaluating the difference of distances between the position of each antenna and every point of the simulated scene. This difference of paths can be converted into a differential phase value. This technique presents one great advantage: it is not necessary to calculate the reflectivity values (facets model of previous sections).

However, if a real interferogram must be simulated, it is not enough to compute the range distances; realistic noise sources must be considered [2]. The simulation of these sources of errors is based on the coherence $\gamma$, which is defined as the cross correlation between the received fields from a same 
point in the observed scene. The analytical development of the coherence results in the following expression for its absolute value:

$$
\begin{aligned}
& |\gamma|=\frac{1}{1+S N R^{-1}} \cdot\left(1-\frac{p}{\lambda} \frac{B_{n}}{r} \frac{\delta_{r}}{\operatorname{tg} \theta_{L}}\right) \cdot\left(1-\frac{\Delta f_{c}}{B_{a z}}\right) \\
& \operatorname{sinc}\left(\frac{\Delta \mathrm{x}}{\delta_{x}}\left(1-\frac{\Delta f_{c}}{B_{a z}}\right)\right) \cdot \sin c\left[\frac{\Delta y}{\delta_{y}}\left(1-\frac{p}{\lambda} \frac{B_{n}}{r} \frac{\delta_{r}}{\operatorname{tg} \theta_{L}}\right)\right]
\end{aligned}
$$

where the most important noise sources have been taken into account: the signal-to-noise ratio $S N R$, the azimuth and ground-range mis-registration ( $\Delta x$ and $\Delta y$ ), the azimuth and ground-range resolution $\left(\delta_{x}\right.$ and $\delta_{y}$ ), the normal baseline $B_{n}$, the baseline mode $p$, the slant-range resolution $\delta_{r}$, the Doppler centroid difference between channels $\Delta f_{c}$ and the azimuth bandwidth $B_{a z}$. The local terrain slope is included in $\theta_{L}$.

Equation (4) can be employed to simulate the noise for every pixel in order to obtain an interferogram with the same decorrelation statistics as a real one.

\section{RESULTS}

To evaluate the simulator performance some SAR images have been generated. The image in Fig. 3 is obtained with our simulator from a real DEM corresponding to an area of $7 \mathrm{~km}$ x $7 \mathrm{~km}$ near Ascó (Tarragona, Spain). The vertical direction corresponds to the azimuth coordinate, whereas the horizontal axis is the range one. The permitivity map has been also considered constant except for the Ebre river, where low values have been assigned. Geometrical distortions produced by the relief such as foreshortening and layover can be appreciated as well as the effect of speckle.

This image has been compared to an ERS-1 processed SLC, acquired on September 12, 1991. Radiometric values are basically the same, and differences are mainly caused by the use of a constant permitivity map in Fig. 3. Displacements between the two images have been also evaluated, obtaining a range precision better than 2 pixels, which gives an idea of the adopted Earth model precision. In azimuth both images match by adjusting the ERS- 1 mission times.

Precision in the second simulation phase has been evaluated by comparing the impulse response phase generated via the inverse ECSA and the phase map obtained from the use of the analytical SAR impulse response equation. Errors have resulted to be under $0.02 \mathrm{rad}$ if zero Doppler deviation is assumed, and for a Doppler centroid of $125 \mathrm{~Hz}$ it maintains under $0.04 \mathrm{rad}$, what validates the use of this simulator as an impulse response generator.

With respect to the interferogram simulator, it has been evaluated by means of airborne data acquired by the DLR's E-SAR system [6]. After geocoding X-band data to a UTM grid, a DEM of the testsite of Oberfaffenhofen (Germany) is available as an input for the simulator. The result is a realistic interferogram, comparable to the real one, whose fringes contain the major sources of noise.

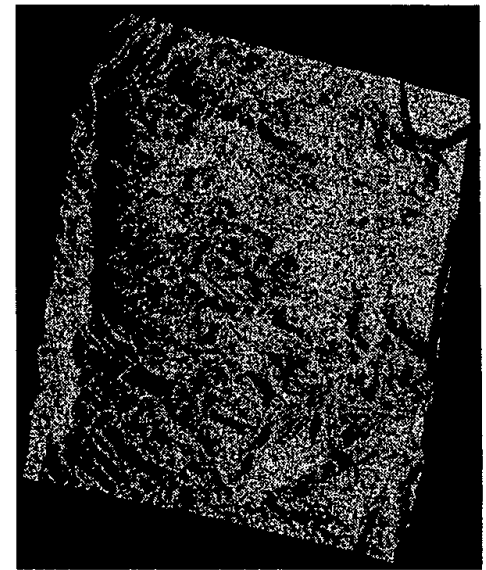

Fig. 3. Simulated SAR image of the zone of Ascó

\section{CONCLUSIONS}

A complete SAR signal simulator frame has been presented. It can be applied in the frame of satellite missions as well as airborne sensors, with the correct geometric and electric features for each case. Moreover, it is devoted not only to generate information with respect to reflectivity, but also to compute realistic interferograms including the main factors, what makes the simulator a powerful tool to design the parameters for topographic reconstruction.

\section{ACKNOWLEDGMENTS}

The authors thank the European Space Agency (ESA), the German Aerospace Center (DLR) and the Cartographic Institute of Catalonia (ICC) for providing the data used in this study. This work has been supported by the Comissionat per Universitats $i$ Recerca (CIRIT) and the Spanish Comission for Science and Technology (CICYT) TIC99-1050-C03-01.

\section{REFERENCES}

[1] G. Franceschetti, M. Migliaccio, D. Riccio and G. Schirinzi, "SARAS: A Synthetic Aperture Radar (SAR) Raw Signal Simulator", IEEE Trans. on Geosc. and Rem. Sens. (TGRS), pp. 110-123, January 1992.

[2] Jan O. Hagberg and Lars M. H. Ulander, "On the Optimization of Interferometric SAR for Topographic Mapping", IEEE TGRS, vol. 31, pp. 303-306, 1993.

[3] A. Moreira, J. Mittermayer, R. Scheiber, "Extended Chirp Scaling Algorithm for Air- and Spaceborne SAR Data Processing in Stripmap and ScanSAR Imaging Modes", IEEE TGRS, vol. 34, September 1996.

[4] J. P. Snyder, "Map Projections used by the US Geological Survey", Washing. Geol. Survey $1983,2^{\text {nd }} \mathrm{Ed}$.

[5] D. Stevens, I. Cumming, A. Gray, "Options for Airborne Interferometric SAR Motion Compensation", IEEE TGRS, vol. 33, n², March 1995.

[6] R. Horn, "The DLR Airborne SAR Project E-SAR", IGARSS'96. 